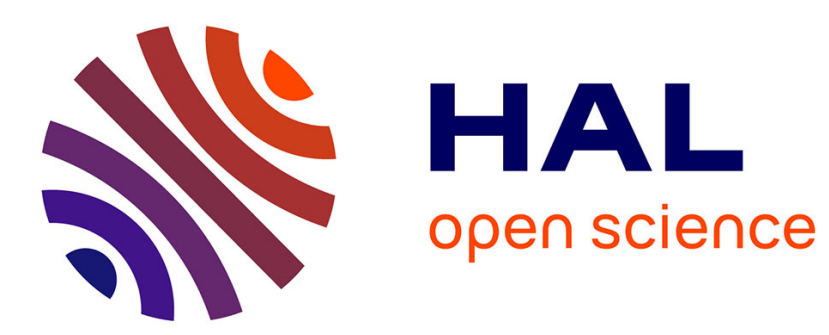

\title{
Solution of static reduced decoupling problem for linear systems \\ Jean François Lafay
}

\section{To cite this version:}

Jean François Lafay. Solution of static reduced decoupling problem for linear systems. 2013. hal00948657

\section{HAL Id: hal-00948657 \\ https://hal.science/hal-00948657}

Preprint submitted on 20 Feb 2014

HAL is a multi-disciplinary open access archive for the deposit and dissemination of scientific research documents, whether they are published or not. The documents may come from teaching and research institutions in France or abroad, or from public or private research centers.
L'archive ouverte pluridisciplinaire HAL, est destinée au dépôt et à la diffusion de documents scientifiques de niveau recherche, publiés ou non, émanant des établissements d'enseignement et de recherche français ou étrangers, des laboratoires publics ou privés. 


\title{
Solution of Static Reduced Decoupling Problem for Linear Systems
}

\author{
Jean-François Lafay* \\ In memoriam A.N. Herrera H. \\ * Institut de Recherche en Communications et Cybernétique de Nantes, \\ IRCCyN (UMR CNRS 6597), \\ Ecole Centrale de Nantes, France.
}

\begin{abstract}
We propose a structural solution by non-regular static state feedback to the diagonal, or row by row decoupling problem for linear systems. Without being completely general, this solution concerns the reduced Morgan's problem, that is we do not want it increases the essential orders of the open loop system. The solution that we propose relies on properties between some partial infinite structures extracted from well chosen interactors and controllability indices of a specific shifted system. To my knowledge, there is at this date only very partial solutions to this problem deemed structurally very difficult.
\end{abstract}

Keywords: linear systems, non regular control, state feedbacks, diagonal decoupling, structural properties.

\section{INTRODUCTION}

Diagonal decoupling by a static state feedback, $u=F x+$ $G v$, or Morgan's Problem, is a very difficult control problem when $G$ is not invertible. Such non regular feedbacks correspond to a loss of inputs for the controlled system. They can transform all invariant structures underlying the regular solutions to control problems. The challenge is to find new structures that will allow us to solve Morgan's Problem, knowing that non regular static solutions can exist even when there is none with regular laws. The non-regular diagonal decoupling by dynamic state feedback is much simpler as by static state feedback. It was solved by Dion and Commault [1988] due to new invariants, the essential orders. Non regular decoupling always comes down to a problem of increasing structures. The relative simplicity of the dynamic case comes from the fact that the essential orders are the minimum infinite structure to achieve for decoupling and that it is always possible to get it if there is a solution. The reason is that, to make this increase, dynamic feedback uses dynamics (integrators) that are external to the system. This is no more the case for static feedbacks that must only use internal dynamics. We consider here the Static Reduced Morgan's Problem (SRMP), say the static decoupling without increasing the essential orders: it provides insight into the complex mechanisms of structural changes by non-regular controls and, from the practical point of vue, this restricted problem is not whitout interest because it is necessary that a dynamic solution exists, (therefore maintaining the essential orders), to hope to find a static one. There are so far only very partial results for the SRMP: when it is suffisant to increase only one element of the infinite structure (Herrera H and Lafay [1993]), or for trivial internal structures, (Zagalak et al. [1998]), (Lafay [2013]). The specific difficulties of SRMP are of two kinds: firstly, although their number is the same and is given by the necessary and sufficient condition of the dynamic problem, the increases of infinite structure for solving SRMP depend on the order of the outputs of the system, while knowing that the sum of the sizes of these increases does not depend of this order. This first lock has been lift in Lafay [2013], where it is proved that there is a unique "minimal list of decoupling indices" such that if SRMP has a static solution, one solution exist with this minimal indices. Secondly we must take into account internal couplings which are unobservable from the outputs to be decoupled. For that, we develop a non trivial general formulation of SRMP inspired by (Herrera $\mathrm{H}$ and Lafay [1993]). The general solution of SRMP relies on the controllability of a well defined shifted system .

\section{NOTATIONS AND BACKGROUND}

\subsection{Notations}

Let $\Sigma(C, A, B)$, denoted shortly $\Sigma$, a linear system whose state is supposed to be measured or reconstructible:

$$
\Sigma\left\{\begin{array}{l}
\dot{x}(t)=A x(t)+B u(t) \\
y(t)=C x(t)
\end{array}\right.
$$

$x \in \mathcal{X} \subset \mathbb{R}_{n}, u \in \mathcal{U} \subset \mathbb{R}_{m}$ and $y \in \mathcal{Y} \subset \mathbb{R}_{p}$ are outputs to be controlled. We suppose $B$ monic and $C$ epic. $\Sigma$ is controllable, right invertible and without finite zeros. This last assumption implies that $\mathcal{V}^{*}=\mathcal{R}^{*}, \mathcal{V}^{*}$ and $\mathcal{R}^{*}$ being the supremal $(A-B)$ invariant and the supremal controllability subspaces in the kernel of $C$ (finite zeros concern only internal stability, which is not adressed here). A set of $\mathrm{p}$ elements is noted $\{\bullet\}_{p}$. Polynomial and rational objects in variable $\mathrm{s}$ are respectively noted $\bullet[s]$ and $\bullet(s)$. We note $\partial p[s]$ the degree of a polynomial and $\partial_{c_{i}} M[s]$ the highest degree of the $i$ th column of matrix $\mathrm{M}[\mathrm{s}]$. Derivative of order $n$ of function $f(t)$ is noted $f^{(n)}(t)$. $\mathbb{I}_{n}$ represents the identity matrix of order $n$ and $\operatorname{diag}\left\{a_{i}\right\}_{p}$ the $p \times p$ matrix which diagonal terms are $a_{i}$, for $i=1, \ldots, p$. 
Definition 1. The interactor of $\Sigma$, (Wolovich and Falb [1976]), is the unique $p \times p$ triangular and non-singular polynomial matrix $\Phi[s]=\left[\varphi_{i, j}[s]\right]$ such that there exists a biproper $m \times m$ (non unique) matrix $B(s)$ satisfying : $T(s)=C\left(s I_{n}-A\right)^{-1} B=\left[\Phi^{-1} 0\right] B(s)$ where:

- $\varphi_{i, i}=s^{d_{i}}, i=1, \ldots, p, d_{i}$ being positive integers

- $\varphi_{i, j}$ is zero or $\varphi_{i, j} / s^{d_{i}}$ is divisible by $s, \forall j>i$.

The interactor is invariant under the action of the group $(T, F, G)$, where $T$ and $G$ are changing of bases on $\mathcal{X}$ and $\mathcal{U}$, and $F$ is a state feedback. Without any loss of generality, we can suppose that it is row reduced (r.r.), (Lafay et al. [1992]).

Four lists of integers characterize partially the structure of $\Sigma$, (see Morse [1973],Commault et al. [1986]):

- $\left\{c_{i}\right\}_{m}:$ controllability indices of $(A, B)$,

- $\left\{n_{i}^{\prime}\right\}_{p}: I_{4}$ Morse's list of the orders of the zeroes at infinity. If the interactor of $\Sigma$ is r.r, $d_{i}=n_{i}^{\prime}, i=1, \ldots, p$.

- $\left\{\sigma_{j}\right\}_{m-p}: I_{2}$ Morse's list providing the structure of $\mathcal{R}^{*}$,

- $\left\{n_{i e}\right\}_{p}:$ the $p$ essential orders of the outputs $y_{i}(t)$ : $n_{i e}=\partial_{c_{i}} \Phi[s], i=1, \ldots, p$, .

These lists are invariant under the action of the group $(T, F, G, \Pi)$, where $\Pi$ is a permutation of the outputs of $\Sigma$ and $G$ is invertible (Herrera $\mathrm{H}$ et al. [1997]).

An extended system $\Sigma^{e}\left(C_{e}, A, B\right)$ is defined in Herrera $\mathrm{H}$ et al. [1997] by adding $m-p$ ficticious outputs, to reflects the structure of $\mathcal{R}^{*}$ taking:

$$
C_{e}=\left[\begin{array}{cc}
C & (0)_{p \times \sum_{j=1}^{m-p} \sigma_{j}} \\
(0)_{(m-p) \times \sum_{i=1}^{p} n_{i}^{\prime}} & \left.\operatorname{diag}\left\{\begin{array}{cccc}
1 & 0 & \ldots & 0
\end{array}\right]_{1 \times \sigma_{j}}\right\}_{m-p}
\end{array}\right]
$$

Proposition 1. The $m \times m$ interactor of $\Sigma^{e}$, called "extended interactor of $\Sigma$ ", has the structure:

$$
\Phi^{e}[s]=\left[\begin{array}{cc}
\Phi_{1}^{e}[s] & (0) \\
\Phi_{2}^{e}[s] & \Phi_{3}^{e}[s]
\end{array}\right]=\left[\varphi^{e}{ }_{i, j}[s]\right], \text { where }
$$

- $\Phi^{e}[s]$ is row reduced

- $\Phi_{1}^{e}[s]$ is the $p \times p$ interactor of $\Sigma$, then $\varphi_{i, i}^{e}=s^{n_{i}^{\prime}}, i=$ $1, \ldots p$,

- $\Phi_{3}^{e}[s]=\operatorname{diag}\left\{s^{\sigma_{1}}, \ldots, s^{\sigma_{m-p}}\right\}$, and

- Polynomials $\left[\varphi^{e}{ }_{i, j}[s]\right]=\sum_{j=l}^{h} \alpha_{j} s^{j}$ of $\Phi_{2}^{e}[s]$ are zero or verify: $n_{j}^{\prime}+1 \leq l$ and $h \leq \sigma_{i}-1$.

Factorize $\Phi[s]$ as $\Phi[s]=W(s) \operatorname{diag}\left\{s^{n_{i e}}\right\}_{p} . \quad W(s)$ is a proper matrix, of rank at infinity $k$, called "proper part of $\Phi[s] . "$ (Dion and Commault [1988]) :

Proposition 2. There exists output's permutation(s) П such that the $p \times p$ interactor $\Phi_{\Pi}$ of $\Sigma_{\Pi}(\Pi C, A, B)$ has the structure:

$$
\Phi_{\Pi}=\left[\begin{array}{lc}
\Phi_{\Pi, 1}[s] & (0) \\
\Phi_{\Pi, 2}[s] & \Phi_{\Pi, 3}[s]
\end{array}\right]=\left[\varphi_{\Pi i, j}[s]\right], \text { where }
$$

- The $k \times k$ polynomial matrix $\Phi_{\Pi, 3}[s]$ is the diagonal matrix $=\operatorname{diag}\left\{s^{n_{j e}}\right\}_{k}$, the $k$ integers $n_{j e}$ being the essential orders of the $k$ last outputs of $\Sigma_{\Pi}$,

- Non zero elements of the infinite structure of the proper part of $\Phi_{\Pi}$ are given by the list $\left\{\delta_{i}\right\}_{p-k}=$ $\left\{n_{i e}-f_{i}\right\}_{p-k}$, where $f_{i}=\partial \varphi_{\Pi_{i, i}}[s]$ for $i=1, \ldots, p-k$.

- $\left\{\delta_{i}\right\}_{p-k}$ is the list of decoupling indices of $\Sigma_{\pi}$,
- There are always, among permutations $\Pi$, some permutations $\Pi_{m}$ such that the list $\left\{\delta_{i}\right\}_{p-k}$ is the unique minimal list $\left\{\Delta_{i}\right\}_{p-k}$ of decoupling indices of $\Sigma$,

- Note that in general $\Phi_{\Pi}$ (and $\Phi_{\Pi_{m}}$ ) are no longer r.r.

The minimal list of decoupling indices, is defined in (Lafay [2013]) using the notion of "minor" list:

Definition 2. Let two lists of integers $\left\{\delta_{i}\right\}_{k 1}$ and $\left\{\gamma_{i}\right\}_{k 2}$ such that $\sum_{i=1}^{k 1} \delta_{i}=\sum_{i=1}^{k 2} \gamma_{i}$. Note $\left\{\hat{\delta}_{i}\right\}_{\text {sup }_{i}}$ and $\left\{\hat{\gamma}_{i}\right\}_{s u p \gamma_{i}}$ their dual lists. $\left\{\delta_{i}\right\}_{k 1}$ "minore" the list $\left\{\gamma_{i}\right\}_{k 2}$ if, for $i=1, . ., \sup \left(\sup _{j}\left(\delta_{j}\right), \sup _{j}\left(\gamma_{j}\right)\right)$, we have:

$$
\sum_{j=1}^{i} \hat{\delta}_{j} \leq \sum_{j=1}^{i} \hat{\gamma}_{j}
$$

This list $\left\{\Delta_{i}\right\}_{p-k}$ and an algorithmic procedure to obtain it are presented in Appendix A in a better way as in Lafay [2013].

\subsection{Impact of a permutation $\Pi$ on the extended interactor}

We consider the extended interactors of $\Sigma(C, A, B)$ and $\Sigma_{\Pi}(\Pi C, A, B)$. We note $\Phi[s]=\left[\varphi_{i, j}\right]$, and $\Phi^{e}[s]$ is structured as in (3).

Property 1. Let a right invertible system $\Sigma$. Let $\Pi$ any permutation matrix acting on the outputs of $\Sigma$. The extended interactor of $\Sigma_{\Pi}$ is given by

$$
\Phi_{\Pi}^{e}[s]=\left[\begin{array}{cc}
\Phi_{\Pi, 1}^{e}[s] & (0) \\
\Phi_{\Pi, 2}^{e}[s] & \Phi_{\Pi, 3}^{e}[s]
\end{array}\right], \text { where }:
$$

- $\Phi_{\Pi, 1}^{e}[s]=\Phi_{\Pi}[s]=B_{1}(s) \Phi[s] \Pi^{-1}$, where $\Phi_{\Pi}[s]$ is the interactor of $\Sigma_{\Pi}$ and $B_{1}(s)$ is a biproper $p \times p$ matrix, - $\Phi_{\Pi, 2}^{e}[s]=B_{2}(s) \Phi_{2 e}[s] \Pi^{-1}$, where $B_{2}(s)$ is a proper $(m-p) \times p$ matrix, which can add polynomials to whose of $\Phi_{2}^{e}[s]$ but, in each column $i$, these polynomials are all of degree less than or equal to $n_{i e}$.

- $\Phi_{\Pi, 3}^{e}[s]=\Phi_{3}^{e}[s]$

The proof is based on the properties of biproper transformations. It is not given here for sake of shortness.

Remark 1. Let us consider equations (3) and (6). Suppose that, for $i=1, \ldots p-k$, one keeps in the ith columns of $\Phi_{2}^{e}[s]$ and $\Phi_{\Pi, 2}^{e}[s]$ only monomials of degree higher than $n_{i e}$ : then, the so "truncated" matrices $\Phi_{2}^{e, t}[s]$ and $\Phi_{\Pi, 2}^{e, t}[s]$ satisfy

$$
\Phi_{\Pi, 2}^{e, t}[s]=\Phi_{2}^{e, t}[s] \Pi^{-1}
$$

\subsection{On the model of the system}

All the transformations involved to obtain interactors or extended interactors correspond to left biproper transformations which can be globally realized by a regular state feedback $\left(F_{m}, G_{m}\right),\left(G_{m}\right.$ invertible), on $\Sigma$ or $\Sigma^{e}$, (Hautus and Heymann [1978]). Then they did not affect the static decouplability of $\Sigma$ and we can suppose that the transfer matrix $T_{m}(s)$ of $\Sigma_{m}$ is $\Phi_{m}^{-1}(s)$. We note $\Sigma_{m}\left(C_{m}, A_{m}, B_{m}\right)$, where $A_{m}=A+B F_{m}, B_{m}=B G_{m}$ and $C_{m}=\Pi_{m} C, F_{m}$ and $G_{m}$ being a regular state feedback . In all what follows it will be assumed, unless explicitly mentioned, that $\Sigma$ is already structured as $\Sigma_{m}$, 
(Proposition 2), with $\left\{\Delta_{i}\right\}_{p-k}=\left\{\delta_{i}\right\}_{p-k}$. To summarize, the extended interactor $\Sigma_{m}^{e}$ is :

$$
\Phi_{m}^{e}[s]=\left[\varphi_{m i, j}^{e}[s]\right]=\left[\begin{array}{c|c|c}
\Phi_{m 1,1}^{e} & (0) & (0) \\
\hline \Phi_{m 1,2}^{e} & \Phi_{m 1,3}^{e} & (0) \\
\hline \Phi_{m 2,1}^{e} & \Phi_{m 2,2}^{e} & \Phi_{m 2,3}^{e}
\end{array}\right]
$$

where the $p \times p$ interactor of $\Sigma_{m}$ is:

$$
\Phi_{m}[s]=\left[\begin{array}{c|c}
\Phi_{m 1,1}^{e} & (0) \\
\hline \Phi_{m 1,2}^{e} & \Phi_{m 1,3}^{e}
\end{array}\right]
$$

- $\Phi_{m 1,3}^{e}=\operatorname{diag}\left\{n_{i e}\right\}_{k}$, where $i=p-k+1, \ldots, p, n_{i e}$ are the essential orders of the $k$ last outputs of $\Sigma_{m}$.

- $\Phi_{m 1,2}^{e}$ is a $p-k \times k$ matrix with $\partial_{c_{i}} \Phi_{m 1,2}^{e}=n_{i e}$,

- $\Phi_{m 1,1}^{e}$ is a $p-k \times p-k$ matrix with: $\varphi_{m i, i}^{e}=s^{f_{i}}$, polynomials $\varphi_{m i, j}^{e}=0$ for $j>i$, and for $i<j$ the non null polynomials $\varphi_{m i, j}^{e}$ are such that $f_{j}+$ $1 \leq \partial_{\min } \varphi_{m i, j}^{e}$ and $\partial_{\max } \varphi_{m i, j}^{e} \leq n_{j e}-1$,

- the list $\left\{n_{i e}-f_{i}\right\}_{p-k}=\left\{\Delta_{i}\right\}_{p-k}$, where $\left\{\Delta_{i}\right\}_{p-k}$ is the unique minimal list of decoupling indices of $\Sigma$,

- $\Phi_{m 2,3}^{e}$ is the $m-p \times m-p$ diagonal matrix $s^{\sigma_{i}}$,

- non null polynomials of $\Phi_{m 2}^{e}=\left[\Phi_{m 2,1}^{e} \mid \Phi_{m 2,2}^{e}\right]$ are such that $f_{j}+1 \leq \partial_{\min } \varphi_{m i, j}^{e}$ for $i \leq p$.

- Note that, for $i \leq p, \partial_{\max } \varphi_{m i, j}^{e}$ can be greater than $n_{j e}$.

\section{MORGAN'S PROBLEM}

\subsection{Formulation of the problem and consequences}

The static diagonal decoupling without stability of $\Sigma$, or Static Morgan's Problem, can be stated as follows: Given $\Sigma$ described by (1), does it exists a static state feedback $u=F x+G v=F x+G_{1} v_{1}+\ldots+G_{p} v_{p}, v_{i} \in \mathcal{U}$ and $G_{i} \in \mathbb{R}^{m \times 1}$ such that, for any $i \in 1,2, \ldots, p, v_{i}$ controls the scalar output $y_{i}$ without affecting the $p-1$ other outputs $y_{j}$ ? If such a feedback exists, the transfer matrix $T_{F G}(s)$ of the closed loop system $\Sigma(C, A+B F, B G)$ is $T_{F G}(s)=\operatorname{diag}\left\{s^{-r_{1}}, \ldots, s^{-r_{p}}\right\}, r_{i} \in \mathbb{R}$. If $G$ is regular (square and invertible) this problem has a solution if and only if the ordered lists $\left\{n_{i}^{\prime}\right\}_{p}$ and $\left\{n_{i e}\right\}_{p}$ are the same. So, the interactor of $\Sigma$ is diagonal (Commault et al. [1986]). If $\left\{n_{i}^{\prime}\right\}_{p} \neq\left\{n_{i e}\right\}_{p}, \Sigma$ will be decouplable if and only if it is possible to increase the structure at infinity so that it matches to (new) essential orders. This modification can only be achieved by a non regular feedback, that is with a loss of inputs. With such controls, the list of essential orders is not always the minimal infinite structure to reach for the static decoupling.

Static Restricted Morgan's Problem, (SRMP), is the particular case of Static Morgan's Problem where the essential orders of $\Sigma$ should be the infinite structure of the decoupled system. SRMP is not without interest because, if static decoupling is only possible with a infinite structure "bigger" than the original essential orders, $\Sigma$ can always be decoupled without modification of these initial essential orders by a non regular dynamic state feedback. More details on that analysis can be found in Herrera $\mathrm{H}$ and Lafay [1993]. Finally, as any non regular decoupling reduces to increase infinite structure, the solution will be based on a theorem of Loiseau [1988] on modifying this structure via non-regular state feedbacks that we remind now:
Theorem 1. Let a linear system for which $\left\{n_{i}^{\prime}\right\}$ is the infinite structure and $\left\{\sigma_{i}\right\}$ the $I_{2}$ Morse's list. Let $\left\{p_{i}\right\}$ a given list of integers. Note $\left\{v_{i}\right\},\left\{\alpha_{i}\right\}$ and $\left\{\pi_{i}\right\}$ the dual lists of, respectively, $\left\{n_{i}^{\prime}\right\},\left\{\sigma_{i}\right\}$ and $\left\{p_{i}\right\}$. Let $\left\{\Gamma_{i}\right\}$ the list obtained by arranging the differences $\left(\pi_{i}-v_{i}\right)$ in a non increasing order. Then, there exists a static state feedback such that the structure at infinity of the closed loop system is the list $\left\{p_{i}\right\}$ if and only if:

$$
\begin{gathered}
v_{1}-v_{i} \geq \pi_{1}-\pi_{i}, \forall i \geq 1, \\
\sum_{i=1}^{j} \alpha_{i} \geq \sum_{i=1}^{j} \Gamma_{i}, \forall j \geq 1 .
\end{gathered}
$$

Before addressing the static problem, let us consider now the dynamic solution of decoupling:

\subsection{The Dynamic Morgan's Problem}

Proposition 3. (Dion and Commault [1988]). The Dynamic Morgan's Problem is solvable if and only if $\Sigma$ is right invertible and $m-p \geq p-k, k$ being the rank at infinity of the proper part of the interactor. Essential orders can always be not modified and such minimal solutions need $\sum_{i=1}^{p} n_{i e}-\sum_{i=1}^{p} n_{i}^{\prime}$ integrators.

The dynamic solution consists in bringing $\Sigma$ in the form $\Sigma_{\Pi}$ (Proposition 2). The list $\left\{\delta_{i}\right\}_{p-k}=\left\{n_{i e}-f_{i}\right\}_{p-k}$ is not necessarily the list of minimal decoupling indices. Dynamic decoupling amounts to annihilating the indices $\left\{\delta_{i}\right\}_{p-k}$ applying the following iterative procedure for $i=$ $1,2, \ldots, p-k$ :

- for $i=1, u_{1}$ is replaced by an external chain of $\delta_{1}$ integrators controlled by an entry of $\mathcal{R}^{*}$, for instance $v_{1}=u_{p+1}$. This external chain is independant of the $(m-p)$ chains of $\sigma_{i}$ integrators of $\mathcal{R}^{*}$. This substitution amounts to multiply the first row of (4) by $s^{\delta_{1}}$. So $\partial \varphi_{\Pi_{1,1}}$ becomes $n_{1 e}$ and it is possible, by a left biproper operation, to eliminate all the other polynomials of the first column of $\Phi_{\Pi}$.

- we successively made the same operation for the $p-k-1$ other rows of $\Phi_{\Pi, 1}$, taking at each step a different entry $v_{i}$ of $\mathcal{R}^{*}$. This is possible because $m-p \geq p-k$.

The final interactor is the $p \times p$ matrix $\operatorname{diag}\left\{s^{n_{i e}}\right\}$ and the system with entries $\left\{v_{1}, v_{2}, \ldots, v_{p-k}, u_{p-k+1}, \ldots, u_{p}\right\}$ is regularly decouplable. So we need $p-k$ chains of independant integrators coming from a dynamic extension of dimension $\sum_{i=1}^{p} n_{i e}-\sum_{i=1}^{p} n_{i}^{\prime}$. This dynamic procedure only require that the number of independent entries of $\mathcal{R}^{*}$ is at least $p-k$. The global number of integrators wich are necessary to decouple does not depend of the permutation $\Pi$ but, even if the list of decoupling indices depends of it. This remark point out one of the main difficulties of Morgan's problem. Another difficulty is the possible existence of couplings between $\mathcal{R}^{*}$ and the rest of the system that are not taking into account by the dynamic solution.

\section{THE SOLUTION OF SRMP}

Let $\Sigma_{m}\left(C_{m}, A_{m}, B_{m}\right)$ as described in subsection 2.3. We note $B_{m}=\left[B_{b}\left|B_{s}\right| B_{r}\right]$, where $B_{b}=\left[b_{1}, \ldots, b_{p-k}\right], B_{s}=$ 
$\left[b_{p-k+1}, \ldots, b_{p}\right]$ and $B_{r}=\mathcal{B} \cap \mathcal{R}^{*}=\left[b_{p+1}, \ldots, b_{m}\right]$. The associated entries are $u(t)=\left[u_{b}(t)\left|u_{S}(t)\right| u_{r}(t)\right]^{T}$. Define the $m \times m$ polynomial matrix $\Phi_{m}^{S, e}[s]$ by:

$$
\Phi_{m}^{S, e}[s]=\left[\begin{array}{cc}
\Phi_{m 1}^{S, e}[s] & (0) \\
\Phi_{m 2}^{S, e}[s] & \Phi_{m 3}^{S, e}[s]
\end{array}\right], \text { where }
$$

- $\Phi_{m 1}^{S, e}[s]=\operatorname{diag}\left\{s^{n_{i e}}\right\}_{p}$

- $\Phi_{m 3}^{S, e}[s]=\operatorname{diag}\left\{s^{\sigma^{i}}\right\}_{m-p}$

- $\Phi_{m 2}^{S, e}[s]$ is deduced from the $m-p \times p$ matrix $\Phi_{m 2}^{e}[s]$ $=\left[\Phi_{m 2,1}^{e} \mid \Phi_{m 2,2}^{e}\right]$ by eliminating, in each column $i=$ $1, \ldots, p$, all monomials of degree less than or equal to $n_{i e}$ by a left biproper transformation.

- $\Phi_{m}^{S, e}[s]$ has the structure of an interactor.

\subsection{Shifted system}

Definition 3. The shifted system $\Sigma_{m}^{S}\left(C_{m}^{S}, A_{m}^{S}, B_{m}^{S}\right)$ associated with $\Sigma_{m}$ is the invertible system of which $\Phi_{m}^{S, e}[s]$, equation (12), is the extended interactor.

Let us extend the state space $\mathcal{X}$ of $\Sigma_{m}$ by adding an extermal dynamic state extension $\mathcal{X}_{a}$, of dimension $n_{a}=\sum_{i=1}^{p-k} \Delta_{i}$, composed of $p-k$ independant controllable and observable chains of integrators of lenghts $\Delta_{1}, \ldots, \Delta_{p-k}$ with entries $w_{1}(t), \ldots, w_{p-k}(t)$ in $\mathcal{U}_{a}$ and outputs $z_{1}(t), \ldots, z_{p-k}(t)$ in $\mathcal{Y}_{a}$. Noting $\oplus$ the external direct sum of subspaces, the state, control and output spaces of $\Sigma_{m}$ completed with this dynamic extension are $\mathcal{X}_{S}=\mathcal{X} \oplus$ $\mathcal{X}_{a}, \mathcal{U}_{S}=\mathcal{U} \oplus \mathcal{U}_{a}$ and $\mathcal{Y}_{S}=\mathcal{Y} \oplus \mathcal{Y}_{a}$.

The shifted system is obtained by replacing the entries $u_{b}(t)$ of $B_{b}$ by $z_{i}(t)$ for $i=1, \ldots, p-k$. The state space realization $\left(C_{m}^{S}, A_{m}^{S}, B_{m}^{S}\right)$ of $\Sigma_{m}^{S}$ is:

$$
A_{m}^{S}=\left[\begin{array}{c|c|c|c}
A_{m} & A_{1}^{S} & \ldots & A_{p-k}^{S} \\
\hline(0) & J_{\Delta_{1}} & (0) & (0) \\
\hline(0) & (0) & \ddots & (0) \\
\hline(0) & \ldots & (0) & J_{\Delta_{p-k}}
\end{array}\right] \text { where : }
$$

- for $i=1, \ldots, p-k, J_{\Delta_{i}}$ is the upper $\Delta_{i} \times \Delta_{i}$ Jordan block and the $n \times \Delta_{i}$ matrices $A_{i}^{S}$ are given by $A_{i}^{S}=$ $\left[b_{i(n \times 1)} \mid(0)_{n \times \Delta_{i}-1}\right]$.

$$
B_{m}^{S}=\left[\begin{array}{c|c|c}
(0)_{(n \times p-k)} & B_{s(n \times k)} & B_{r(n \times m-p)} \\
\hline B_{a\left(n_{a} \times p-k\right)} & (0) & (0)
\end{array}\right],
$$

where $B_{a}=\left[b_{a, 1}, \ldots, b_{a, p-k}\right], b_{a, i}=\left[\begin{array}{llll}0 & \ldots & 0 & 1\end{array}\right]^{T}$ being $\Delta_{i} \times 1$ vectors.

$$
C_{m}^{S}=\left[C_{m(p \times m)} \mid(0)_{\left(p \times n_{a}\right)}\right] .
$$

Then, as in noted in Subsection 3.2 the interactor of $\Sigma_{m}^{S}$ is given by

$$
\Phi_{m}^{S}[s]=\left[\operatorname{diag}\left\{s^{n_{i e}}\right\}_{p} \mid(0)_{p \times m-p}\right],
$$

and the corresponding extended interactor of $\Sigma_{m}^{S}$ is:

$$
\Phi_{m}^{S, e}[s]=\left[\begin{array}{c|c}
\operatorname{diag}\left\{s^{n_{i e}}\right\}_{p} & (0)_{p \times m-p} \\
\hline \Phi_{m, 2}^{S, e} & \operatorname{diag}\left\{s^{\sigma_{i}}\right\}_{m-p}
\end{array}\right],
$$

where $\Phi_{m 2}^{S, e}[s]$ is deduced from $\Phi_{m 2}^{e}[s]$ by eliminating in each column $j=1, \ldots p$ the monomials of degree less than or equal to $n_{j e}$

\subsection{A convenient formulation of SRMP}

SRMP will be solved if it is possible to replace the $p-$ $k$ independant chains of external integrators solving the dynamic Morgan's problem by $p-k$ independant chains of integrators extracted from $\Sigma$. A chain of lenght $\mathcal{L}$ is a set of $\mathcal{L}$ connected integrators, actived only at its beginning by a function $f_{a}(t)$ to generate a function $f_{e}(t)$, ie $f_{e}^{(\mathcal{L})}(t)=f_{a}(t)$. Let set of $q$ chains of integrators defined by $f_{e}^{i\left(\mathcal{L}_{i}\right)}(t)=f_{a}^{i}(t), i=1, \ldots, q$. For SRMP, we require that each function $f_{a}^{i}(t)$ contains at least one input of the the system. These chains are independant if, $\forall j \neq i$, the term containing the inputs in $f_{a}^{i}(t)$ is not a linear combination of terms containing the inputs in $f_{a}^{j}(t)$, and if $f_{e}^{i}(t)$ is not a linear combination of functions $f_{e}^{j}(t)$. The following lemma characterize internal chains that will increase the infinite structure without changing the essential orders: it is a new formulation for SRMP, generalizing in a non trivial way Lemma 4.2 in (Herrera $\mathrm{H}$ and Lafay [1993])valid when $k=p-1$.

Lemma 1. Let a right invertible system $\Sigma_{m}$ with $\mathcal{R}^{*}=$ $\mathcal{V}^{*}, k$ the rank of the proper part of its interactor and $\left\{\Delta^{i}\right\}_{(p-k)}$ its decouplability indices (say the minimal decoupling indices of $\Sigma$ ). Then, SRMP has a solution if and only if it is possible to extract from $\mathcal{R}^{*} p-k$ independant chains of integrators of lengths $\Delta_{1}, \ldots, \Delta_{p-k}$ described by $f_{e}^{i}(t)^{\left(\Delta_{i}\right)}=f_{a}^{i}(t), i=1, \ldots, p-k$ such that:

(a) The output $f_{e}^{i}(t)$ of each chain of integrators is only function of the vector state space $x(t)$ and these $p-k$ functions are independant,

(b) For $i=1, \ldots, p-k$, the entry $f_{a}^{i}(t)$ of each chain of integrators do not contain derivatives of inputs $u_{j}(t), j=p-k+1, \ldots, p$.

(c) For $i=1, \ldots, p-k$, the entry $f_{a}^{i}(t)$ of each chain of integrators do not contain derivatives of inputs $u_{j}(t)$ of order greather than or equal to $\Delta_{j}$, for all $j=1, \ldots, p-k$.

Proof 1. of Lemma 1 IF. Let a state feedback $(F, G)$ which decouples $\Sigma_{m}\left(C_{m}, A_{m}, B_{m}\right)$. According to Dion and Commault [1988] this feedback is equivalent to the precompensator $C(s)=\left(\mathbb{I}_{m}-F\left(s \mathbb{I}_{n}-A_{m}\right)^{-1} B_{m}\right)^{-1} G=$ $\left[\begin{array}{c}W_{1, m}(s) \\ X(s)\end{array}\right]$, where $X(s)$ is a proper $m-p \times p$ matrix and, noting $d=s^{-1}$, the $p \times p$ proper part $W_{1, m}(s)$ of the interactor of $\Sigma_{m}$ can be written as:

$$
W_{1, m}(s)=\left[\begin{array}{cccccc}
d^{\Delta_{1}} & & & & & \\
\hat{\varphi}_{2,1} & \ddots & & & (0) & \\
& & d^{\Delta_{p-k}} & & & \\
& \left(\hat{\varphi}_{i, j}\right) & \vdots & 1 & & \\
& & \vdots & (0) & \ddots & \\
\hat{\varphi}_{p, 1} & & \hat{\varphi}_{p, p-k} & 0 & \cdots & 1
\end{array}\right] .
$$

$\hat{\varphi}_{i, j}[d]$ are polynomials in $d=s^{-1}$ such that $\partial \hat{\varphi}_{i, j}[d]<$ $\Delta_{j}=n_{j e}-f_{j}$ for $i \leq p-k$.

Matrix $G$ of the feedback $(F, G)$ is obtained (Herrera $\mathrm{H}$ [1992]) by: $G=\lim _{s \rightarrow \infty} W_{1, m}(s)=\left[\begin{array}{l|l}(0)_{p-k \times p-k} & (0) \\ \hline\left(g_{i, j}\right)_{k \times p-k} & \mathbb{I}_{k}\end{array}\right]$ where $g_{i, j} \in \mathbb{R}$. 


$$
u_{i}(t)=\left\{\begin{array}{cc}
F_{i} x(t), & i=1, \ldots, p-k \\
F_{i} x(t)+\sum_{j=1}^{p-k} g_{i, j} v_{j}(t)+v_{i}(t), & i=p-k+1, . ., p
\end{array}\right.
$$

As $\Sigma_{m,(F, G)}\left(C_{m}, A_{m}+B_{m} F, B_{m} G\right)$ is assumed to be decoupled without changing the essential orders, we have:

$$
y_{i}^{\left(n_{i e}\right)}(t)=\left\{\begin{array}{l}
u_{i}^{\left(\Delta_{i}\right)}(t)=v_{i}(t), i=1, \ldots p-k \\
u_{i}^{(0)}(t)=v_{i}(t), i=p-k+1, \ldots, p .
\end{array}\right.
$$

Therefore, equations (20) and (19a) describe $p-k$ chains of lenghts $\Delta_{1}, \ldots, \Delta_{p-k}$ generating independant functions $F_{i} x(t)$ of the state $x(t)$. Moreover, from (19b), these chains are not activated by derivatives of the entries $u_{p-k+1}(t), \ldots, u_{p}(t)$ and for $i$ and $j=1, \ldots, p-k$, the ith chain is not actived by derivatives of $v_{j}(t), i \neq j$. But, by equation $(19 \mathrm{~b})$, if the role of the chains is only to make $\Sigma_{m}$ regularly decouplable, the ith chain can be eventually activated by derivatives of entries $u_{i}(t)$ of order less than $\Delta_{i}$ for $i=1, \ldots, p-k$, because for each chain must generate a function of the state $x(t)$, say: $f_{a}^{i}(t)=f_{e}^{i\left(\Delta_{i}\right)}(t)$ and $f_{a}^{i}(t)=F_{i} x(t)$

According Loiseau [1988], since any increasing of infinite structure can only be done using entries of $\mathcal{R}^{*}$, entries $v_{1}(t), \ldots, v_{p-k}(t)$ should contain independant linear combinations of entries of $\mathcal{R}^{*}$.

(Only if): Assume that these $p-k$ independant chains can be constructed. For $i=1, \ldots, p-k$, each chain is characterized by its lenght $\Delta_{i}$, its entry $f_{a}^{i}(t)$ and its output $f_{e}^{i}(t)$ linked by $f_{e}^{i\left(\Delta_{i}\right)}(t)=f_{a}^{i}(t)$. As $f_{e}^{i}(t)$ is uniquely a function of the state space of $\Sigma_{m}$, we can write $f_{e}^{i}(t)=F_{i} x(t)$, where $F_{i} \in \mathbb{R}^{1 \times n}$, and as the chains are independant, rank $\bar{F}=\left[\begin{array}{c}F_{1} \\ \vdots \\ F_{p-k}\end{array}\right]=p-k$.

Now, consider the entries of the chains. As these chains are extracted fom $\mathcal{R}^{*}$, each function $f_{a}^{i}(t)$ contains at least one linear combination of the entries $u_{p+1}(t), \ldots, u_{m}(t)$ and these linear combinations are independant: this implies that $\operatorname{dim} \mathcal{B} \cap \mathcal{R}^{*} \geq p-k$. This is the necessary and sufficient condition of dynamic decoupling for $\Sigma$. For static decoupling, we must add the condition: $\sum_{i=1}^{p-k} \Delta_{i} \leq$ $\sum_{i=1}^{m-p} \sigma_{i}$, where $\left\{\sigma_{i}\right\}_{m-p}$ is the Morse's list $I_{2}$.

By assumption, no derivative of entries $u_{p-k+1}(t), \ldots, u_{p}(t)$ appears in functions $f_{a}^{i}(t)$. However there may be terms which depend on the state $x(t)$ and/or on entries $u_{p-k+1}(t), \ldots, u_{p}(t)$, and/or, for $i=1, \ldots, p-k$, on derivatives of entries $u_{i}(t)$ of order less than $\Delta_{i}$, (because each function $f_{a}^{i}(t)=f_{e}^{i\left(\Delta_{i}\right)}(t)$ does not include derivative of $u_{i}(t)$ of order greather than or equal to $\Delta_{i}$ from the fact that $f_{e}^{i}=F_{i} x(t)$. So, for $i=1, \ldots, p-k$, the general form of functions $f_{a}^{i}(t)$ is:

$$
\begin{aligned}
f_{a}^{i}(t)=f_{a 1}^{i}( & \left.u_{p+1}(t), \ldots, u_{m}(t)\right) \\
& +f_{a 2}^{i}\left(x(t), u_{p-k+1}(t), \ldots, u_{p}(t)\right) \\
& +\sum_{j=1}^{p-k} f_{a 3, j}^{i}\left(u_{j}^{(1)}(t), \ldots, u_{j}^{\left(\Delta_{j}-1\right)}(t)\right) .
\end{aligned}
$$

$f_{a 1}^{i}$ are nonzero and independant functions.

As $u_{i}^{(j)}(t)=y_{i}^{\left(f_{i}+j\right)}(t)=y_{i}^{\left(n_{i e}-\Delta_{i}+j\right)}(t)$, the Laplace's transform of each function $f_{a}^{i}(t)$ is:

$$
\begin{aligned}
f_{a}^{i}(s)=f_{a 1}^{i}\left(u_{p+1}(s), \ldots, u_{m}(s)\right) & \\
& +f_{a 2}^{i}\left(x(s), u_{p-k+1}(s), \ldots, u_{p}(s)\right) \\
& +\sum_{j=1}^{p-k} \Psi_{i, j}[s] y_{j}(s),
\end{aligned}
$$

where the degree of each polynomial $\Psi_{i, j}[s]$ is less than $n_{j e}$.

We can now define the non regular feedback $u(t)=F x(t)+$ $G v(t)$ by:

$F=\left[\begin{array}{c}\bar{F}_{p-k \times n} \\ \hline 0_{k \times n} \\ \hline F_{0 m-p \times n}\end{array}\right]$ and $G=\left[\begin{array}{c|c}0_{p-k \times k} & 0_{p-k \times p-k} \\ \hline \mathbb{I}_{k} & 0_{k \times p-k} \\ \hline 0_{m-p \times k} & G_{0 m-p \times p-k}\end{array}\right]$.

Here $\operatorname{rank} \bar{F}=p-k$ and $\operatorname{rank} G_{0}=p-k \leq m-p$.

The possibly non regular feedback $\left(F_{0}, G_{0}\right)$ acts only on $\mathcal{R}^{*}$ to create the $p-k$ independant chains of integrators. Noting $B_{r}=\operatorname{Im} B \cap \mathcal{R}^{*}$ and $x_{\mathcal{R}^{*}}(t)$ trajectories in $\mathcal{R}^{*}$, this part of feedback is such that: $F_{0} x_{\mathcal{R}^{*}}(t)+B_{r} G_{0} v_{*}(t)=$ $\left[\begin{array}{c}f_{a 1}^{1}(\bullet) \\ \vdots \\ f_{a 1}^{p-k}(\bullet)\end{array}\right]$, where $v_{*}(t)=\left[\begin{array}{c}v_{p-k+1}(t) \\ \vdots \\ v_{p}(t)\end{array}\right]$.

Then, as $y_{i}^{\left(f_{i}\right)}(t)=u_{i}(t)=f_{e}^{i}(t)$ for $i=1, \ldots, p-k$, we obtain: $\mathcal{L}\left(y_{i}^{\left(n_{i e}\right)}(t)\right)=f_{e}^{i} x(s) s^{\Delta_{i}}=f_{a}^{i}(t)=f_{a 1}^{i}(\bullet)+$ $f_{a 2}^{i}(\bullet)+\sum_{j=1}^{p-k} \Psi_{i, j}[s] y_{j}(s)$ with $\partial \Psi_{i, j}[s]<n_{j e}$. Then, outputs $y(s)$ and new entries $v(s)$ are linked by:

$$
\left[\begin{array}{c|c}
H_{1,1}[s] & (0)_{p-k \times k} \\
\hline H_{2,1}[s] & H_{2,2}[s]
\end{array}\right] y(s)=V(s)=\left[\frac{V_{1}(s)}{V_{2}(s)}\right],
$$

where

1- The $p-k \times p-k$ polynomial matrix $H_{1,1}$ is given by:

$$
H_{1,1}=\left[\begin{array}{ccc}
s^{n_{1 e}}-\Psi_{1,1} & & \\
& \ddots & \left(-\Psi_{i, j}\right) \\
\left(\varphi_{m i, j}^{e}-\Psi_{i, j}\right) & & s^{n_{(p-k) e}}-\Psi_{p-k, p-k}
\end{array}\right]
$$

2- $H_{2,1}=\left[\varphi_{m i, j}^{e}[s]\right]$ is a $k \times p-k$ polynomial matrix 3- $H_{2,2}[s]=\operatorname{diag}\left\{s^{n_{(p-k+1) e}}, \ldots, s^{n_{p e}}\right\}_{k}$.

$$
\begin{aligned}
& \text { 4- } V_{1}(s)=\left[\begin{array}{c}
f_{a 1}^{1}(\bullet)+f_{a 2}^{1}(\bullet) \\
\vdots \\
f_{a 1}^{p-k}(\bullet)+f_{a 2}^{p-k}(\bullet)
\end{array}\right] \text { and } \\
& V_{2}(s)=\left[\begin{array}{c}
v_{p-k+1}(s)=u_{p-k+1}(s) \\
\vdots \\
v_{p}(s)=u_{p}(s)
\end{array}\right] \text {. }
\end{aligned}
$$

As the polynomial of highest degree of each column of $H[s]$ is on the diagonal the matrix, the infinite structure coincides with essential orders. This system is then regularly decouplable without increasing the essential orders.This ends the proof of Lemma 1.

Remark 2. Functions $f_{a}^{i}(t)$ cannot contain derivatives ou entries of $\mathcal{R}^{*}$. If that were the case, for exemple for the first chain, the effective lenght of this chain would be less than $\Delta_{1}$. Indeed, suppose that this chain of lenght $\Delta_{1}$ is activated by $u_{p+1}(t)$ and by $u_{p+j}^{(1)}(t)$, for $j=1, \ldots, m-$ $p$. Then $f_{e}^{1\left(_{1}\right)}(t)=f_{a}^{1}(t)=u_{p+1}(t)+u_{p+j}^{(1)}(t)$ and 
$\left.f_{e}^{1}{ }^{\left(\Delta_{1}-1\right.}\right)(t)=\int f_{a}^{1}(t) d t=\int u_{p+1}(t) d t+\int u_{p+j}^{(1)}(t) d t=$ $f(x(t))+u_{p+j}(t)$. The effective lenght of the chain is $\Delta_{1}-1$.

Remark 3. Inputs $u_{1}(t), \ldots, u_{p-k}(t)$ are suppressed, while inputs $u_{p-k+1}(t), \ldots, u_{p}(t)$ are preserved.

Remark 4. If functions $f_{a}^{i}(t)$ do not include derivatives of inputs $u_{i}(t), i=1, \ldots, p-k$, the system is decoupled.

Lemma 1 will help us to derive conditions on $\Sigma_{m}$ for ensure that such $p-k$ independant decoupling chains exist.

To characterize the maximal lenghts of the chains of $\mathcal{R}^{*}$ which are not actived by derivatives of entries $u_{j}(t)$ of order higher or equal to $\Delta_{j}, j=1, \ldots, p$, it is possible to apply Theorem 4.1 in Herrera $\mathrm{H}$ and Lafay [1993] taking into account the following remark:

Remark 5. The chains of $\mathcal{R}^{*}$ satisfying items (b) and (c) of Lemma1 for $\Sigma_{m}$ are the same as the chains of $\mathcal{R}^{*}$ which are not actived by derivatives of entries $w_{1}, \ldots, w_{p-k}$ $u_{p-k+1}, \ldots, u_{p}$ for $\Sigma_{m}^{S}$.

Theorem 4.1 in Herrera H and Lafay [1993] becomes:

Proposition 4. Let $\Sigma_{m}^{S}$ be given. Let $\left\{c_{i}\right\}_{m}$ the controllability indices of $\left(A^{S}{ }_{m}, B^{S}{ }_{m}\right)$. Then the set $\left\{\alpha_{i}\right\}_{m-p}$ of maximal lenghts of subchains of $\mathcal{R}^{*}$ not activated by derivatives of inputs $w_{1}, \ldots, w_{p-k}, u_{p-k+1}, \ldots, u_{p}$ is

$$
\alpha_{i}=c_{i}, \quad \text { for } i=p+1, \ldots, m \text {. }
$$

\subsection{Main result: the solution of SRMP}

Theorem 2. Let the right invertible system $\Sigma$ be given with $\mathcal{R}^{*}=\mathcal{V}^{*}$ and $k$ the rank at infinity of the proper part of its interactor. Let $\Sigma_{m}$ deducted by regular state feedback from $\Sigma$ such that the infinite structure $\left\{\Delta_{i}\right\}_{(p-k)}$ of the proper part of the interactor of $\Sigma_{m}$ is the minimal list of decoupling indices of $\Sigma$. Let $\left\{\alpha_{i}\right\}_{(m-p)}$ the controllability indices of the entries of $\mathcal{R}^{*}$ for the shifted system $\Sigma_{m}^{S}$ associated wit $\Sigma_{m}$. Then SRMP has a solution if and only if, for all $i \geq 1$,

$$
\sum_{j=1}^{i} \hat{\alpha}_{j} \geq \sum_{j=1}^{i} \gamma_{j}
$$

where $\left\{\hat{\alpha}_{i}\right\}_{\sup \alpha_{i}}$ the dual list of $\left\{\alpha_{i}\right\}_{(m-p)}$ and $\left\{\gamma_{i}\right\}_{\sup \Delta_{i}}$ is the dual list of $\left\{\Delta_{i}\right\}_{(p-k)}$.

\section{Proof 2. Sufficiency:}

Theorem 1 cannot be applied directly because it transforms globally a list $\left\{n_{i}^{\prime}\right\}_{p-k}$ into a list $\left\{n_{i e}\right\}_{p-k}$, and for decoupling, the transformations will be done done term by term. If this is was not the case, the essential orders would be changed. But, as mentionned in Lafay [2013] it suffices to apply Theorem 1 choosing for list $\left\{n_{i}^{\prime}\right\}_{p-k}$ the list $\{1\}_{p-k}$ and try to turn it into the list $\left\{1+\Delta_{i}\right\}_{p-k}$. This amounts to build, from the $m-p$ chains of integrators of lengths $\sigma_{i}$ of $\mathcal{R}^{*}, p-k$ independent chains of lengths $\left\{\Delta_{i}\right\}$. Note that condition (10) is still always true. So there remains only conditions (11).

Let us now return to SRMP. It is sufficient to replace the dynamic extension of Subsection 4.1 by $p-k$ independant chains of lenghts $\left\{\Delta_{i}\right\}_{(p-k)}$ extracted from $\mathcal{R}^{*}$ and satisfying Lemma 1. This is equivalent to create, from $\Sigma_{m}^{S}$, $p-k$ independant chains extracted from $\mathcal{R}^{*}$ not actived by derivatives of entries $w_{1}, \ldots, w_{p-k}, u_{p-k+1}, \ldots, u_{p}$ (cf Remark 5). By Proposition 4, such chains should be built from the maximal subchains of $\mathcal{R}^{*}$ which are not undividually actived by derivatives of $w_{1}, \ldots, w_{p-k}, u_{p-k+1}, \ldots, u_{p}$.

According to the construction of the shifted system, and Remark 5, integers $\left\{\alpha_{i}\right\}_{m-p}$ represent equivalently the maximal lenghts of subchains of $\mathcal{R}^{*}$ not activated by derivatives of inputs $u_{p-k+1}, \ldots, u_{p}$ and for $i=1, \ldots, p-k$ not activated by derivatives of inputs $u_{i}$ of order greather than or equal to $\Delta_{i}$. Applying Proposition 1 as mentionned at the beginning of this proof, we obtain condition (26) which is sufficient to solve SRMP.

Necessity: The necessity of this condition comes from two facts. First: by Proposition 2, if there is a solution to SRMP, there are some which which require only ( $\mathrm{p}-$ $\mathrm{k}$ ) increases of infinite structure. Conditions (11 therefore requires that the minimum list of decoupling indices contain only (p-k)termes. Secondly: the list of minimal decoupling indices contains ( $\mathrm{p}-\mathrm{k})$ terms and "minore" all the other lists of decoupling indices. In other words, if there is no solution for these indices, so with the order of outputs of $\Sigma_{m}$, it does not exist solution for any other list if decoupling indices coming from other permutations of outputs of $\Sigma$. This ends the proof .

Corollary 1. The condition (26) of Theorem 2 is a necessary and sufficient condition for the general Morgan's problem for a system $\Sigma$ when $m-p=k$.

\section{COMPARISONS}

At our knowledge, the more advanced structural results on static Morgan's problem are in Herrera H and Lafay [1993], Zagalak et al. [1998] and Lafay [2013].

\subsection{Herrera $H$ and Lafay [1993]}

In this paper, SRMP is solved when $k=p-1$. The solution was stated as follows, with the notations adopted here:

Theorem 3. Let the right invertible system $\Sigma_{m}$ be given with $\mathcal{R}^{*}=\mathcal{V}^{*}$. Suppose $k=p-1$. Let $\delta_{1}$ be the (nonzero)infinite structure of the interactor $\Pi_{1 m}[s]$, and $\left\{\alpha_{r, i}\right\}_{m-p}$ the controllability indices of the pair $\left(A_{m},\left[B_{s} \mid B_{r}\right]\right)$ related with the columns of $B_{r}$. Then the SRMP has a solution if and only if:

$$
\sum_{j=1}^{m-p} \alpha_{r, j} \geq \delta_{1} \text {. }
$$

Le $\Sigma_{m}=\left(C_{m}, A_{m}, B_{m}\right)$ and note $B_{m}=\left[b_{1}\left|B_{s}\right| B_{r}\right]$ as in Section 4. In this particular case, the list of decoupling indices contains only one term $\delta_{1}=\sum_{i=1}^{p} n_{i e}-\sum_{i=1}^{p} n_{i}^{\prime}$. Then this list is minimal. A static solution will exist if and only if the sum of lenghts of the subchains of $\mathcal{R}^{*}$ not actived by derivatives of entries $\left(u_{2}, \ldots, u_{p}\right)$ is greather than or equal to $\delta_{1}$ which is strictly less than $n_{1 e}$. Consider now the shifted system associated with $\Sigma_{m}$. Following equations (13) and (14), we have:

$$
A_{m}^{S}=\left[\begin{array}{c|c}
A_{m} & A_{1}^{S} \\
\hline(0) & J_{\Delta_{1}}
\end{array}\right] \text { where }
$$


- $J_{\delta_{1}}$ is the upper $\delta_{1} \times \Delta_{1}$ Jordan bloc, and matrix $A_{i}^{S}$ are given by $A_{1}^{S}=\left[b_{1(n \times 1)} \mid(0)_{n \times \Delta_{1}-1}\right]$.

- $B_{m}^{S} 1=$

$$
\begin{aligned}
& {\left[\begin{array}{l|c|c}
(0)_{(n \times 1)} & B_{s(n \times p-1)} & B_{r(n \times m-p)} \\
\hline b_{a\left(\delta_{1} \times 1\right)} & (0) & (0)
\end{array}\right] \text { with } } \\
b_{a}= & {\left[\begin{array}{llll}
0 & \ldots & 0 & 1
\end{array}\right]^{T} . }
\end{aligned}
$$

Let us compare the controllability indices $\left\{\alpha_{i}\right\}_{m-1}$ of the the pair $\left(A_{m},\left[B_{s} \mid B_{r}\right]\right)$ and the controllability $\left\{c^{S}{ }_{i}\right\}_{m}$ of $\Sigma^{S}{ }_{m}$. Using the Brunovsky's procedure, Brunovski [1970], we have $c^{S}{ }_{1} \geq n_{1 e}$, then

- if $\alpha_{i} \leq n_{1 e}$, then $\alpha_{i}=c^{S}{ }_{i+1}$, and

- if $\alpha_{i}>n_{1 e}$, we can have $c^{S}{ }_{i+1}<\alpha_{i}$ but $c^{S}{ }_{i+1}>n_{1 e}$.

Then as $\delta_{1} \leq n_{1 e}$ the two Theorems are equivalents for the existence of a solution for SRMP.

\subsection{Zagalak et al. [1998]}

In this paper, the authors consider systems $\Sigma$ with special conditions on dimensions as $m=2 p$ and $\sum_{i=1}^{p} \delta_{i}=$ $\sum_{i=1}^{p} \sigma_{i}$, and mainly without couplings between $\mathcal{R}^{*}$ and the blocks of infinite structure, ie $\Phi_{m 2}^{e}=\left[\Phi_{m 2,1}^{e} \mid \Phi_{m 2,2}^{e}\right]=[0]$ in the extended interactor of $\Sigma_{m}$. The problem was however difficult althought these assumptions seem very simplistic. Certainly, the authors propose factorizations which must have a link with the minimal list of decoupling indices but, unless I have not properly understood their approach, this structural information does never appear explicitly.

\section{CONCLUSION}

In this paper, we propose a necessary and sufficient conditions for the row by row decoupling problem without modifying the essential orders. Even in this special form, the problem was recognized as structurally difficult. It remains to solve the general problem which is much more difficult because we do not know yet how to define minimal structures for the decoupled system.

\section{Appendix A. MINIMAL LIST OF DECOUPLING INDICES}

We give here the procedure to obtain the minimal list $\left\{\Delta_{i}\right\}_{p-k}$;

Let the interactor $\Phi[s]=\left[\begin{array}{cc}\Phi_{1}[s] & (0) \\ \Phi_{2}[s] & \Phi_{3}[s]\end{array}\right]=\left[\varphi_{i, j}[s]\right]$ of $\Sigma$ that is supposed to be in the form (4).

For columns $1,2, \ldots, p-k$, we note $p_{i e}$ the maximum degree of the $i$ th column of $\Phi_{1}[s], \gamma_{i}=n_{i e}-p_{i e}$ and define the index $\Delta=\min \{\gamma\}_{p-k}$. We will first prove that $\Delta$ does not depend on the permutation $\Pi$ such as the interactor of $\Sigma_{\Pi}$ is under the form (4) and in a second step that it is the smallest decoupling index possible for $\Sigma_{\Pi}$.

According to Proposition 2, the polynomial of highest degree of each column of $\left[\begin{array}{l}\Phi_{1}[s] \\ \Phi_{2}[s]\end{array}\right]$ belongs to $\Phi_{2}[s]$. Let $\varphi_{t, j}[s]$, with $j \leq p-k$ and $t>p-k$, this polynomial for the $j$ th column of $\Phi[s]$. Its degree is $n_{j e}$. Let $\Pi^{-1}$ the permutation of the $t$ th and $j$ th columns of $\Phi[s]$, which corresponds to the permutation $\Pi$ of the $t$ th and $j$ th outputs of (1). $\Phi[s] \Pi^{-1}$ is no longer an interactor.

\section{A.1 $\Delta$ is not modified}

To simplify the notations and without loss of generality, we assume that $j=1$ and $t=p$. Noting (only for the next equation) $\alpha=p-k, \Phi[s] \Pi^{-1}$ is given by:

$\left[\begin{array}{cccc|cccc}0 & 0 & \cdot & \cdot & \cdot & \cdot & 0 & \varphi_{1,1} \\ 0 & \varphi_{2,2} & \cdot & \cdot & \cdot & \cdot & 0 & \varphi_{2,1} \\ \cdot & \cdot & \cdot & \cdot & \cdot & \cdot & \cdot & \cdot \\ 0 & \varphi_{\alpha, 2} & \cdot & \varphi_{\alpha, \alpha} & \cdot & \cdot & 0 & \cdot \\ \hline 0 & \varphi_{\alpha+1,2} & \cdot & \cdot & \varphi_{\alpha+1, \alpha+1} & \cdot & \cdot & \cdot \\ \cdot & \cdot & \cdot & \cdot & 0 & \cdot & \cdot & \cdot \\ 0 & \varphi_{p-1,2} & \cdot & \cdot & 0 & 0 & \varphi_{p-1, p-1} & \cdot \\ \varphi_{p, p} & \varphi_{p, 2} & \cdot & \varphi_{p, \alpha} & 0 & 0 & 0 & \varphi_{p, 1}\end{array}\right]$

We will determine a biproper matrix $B_{\Pi}(s)$ such that $\Phi_{\Pi}[s]=B_{\Pi}(s) \Phi[s] \Pi^{-1}$ is the interactor of $\Sigma_{\Pi}$. First we cancel by a left biproper operation $B_{1}(s)$ the polynomials $\varphi_{j, 1}, j=1, \ldots, p-1$, of the $p$ th column of (A.1). $B_{1}(s)$ always exists since $\partial \varphi_{p, 1}[s]=n_{1 e} \geq \partial \varphi_{j, 1}[s], \forall j$. Choosing

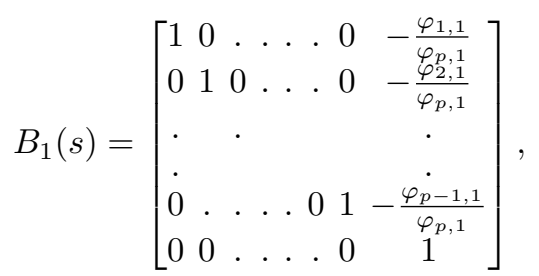

$$
B_{1}(s) \Phi \Pi^{-1}=\left[\begin{array}{cc}
\hat{\Phi}_{1}(s) & (0) \\
\hat{\Phi}_{2}(s) & \hat{\Phi}_{3}[s]
\end{array}\right]=\left[\hat{\varphi}_{i, j}\right]
$$

where $\hat{\Phi}_{1}[s]$ is a $p-k \times p-k$ matrix, and where $\hat{\Phi}_{3}[s]$ and $\Phi_{3}[s]$ differ only by $\hat{\varphi}_{p, p}=\varphi_{p, 1}$ while $^{1} \hat{\varphi}_{p, 1}=s^{n_{p e}}=\varphi_{p, p}$. Except this permutation, the other polynomials of the $p$ th row of $B_{1}(s) \Phi \Pi^{-1}$ are not modified. Note that $\hat{\Phi}_{1}(s)$ and $\hat{\Phi}_{2}(s)$ are no longer necessarily polynomial matrices. Consider now the other columns of $B_{1}(s) \Phi[s] \Pi^{-1}$ :

Lemma 2. For $j=2, \ldots, p-k$, the highest degree the jth columns of $B_{1}(s) \Phi \Pi^{-1}$ and $\Phi[s]$ is not changed and the polynomials of degree $n_{j e}$ are in $\hat{\Phi}_{2}[s]$.

Proof 3. The degrees of columns are the same because $B_{1}(s)$ is biproper. In equation (A.3), $\varphi_{i, j}$ is transformed as $\hat{\varphi}_{i, j}=\varphi_{i, j}-\frac{\varphi_{i, 1}}{\varphi_{p, 1}} \varphi_{p, j}$. For $i$ and $j=1, \ldots, p-k, \partial \varphi_{i, j}<n_{j e}$. As $\partial \varphi_{i, 1}<\partial \varphi_{p, 1}$ and $\partial \varphi_{p, j} \leq n_{j e}$, we have $\partial \frac{\varphi_{i, 1}}{\varphi_{p, 1}} \varphi_{p, j}<$ $n_{j e}$, which ends the proof of Lemma 2 .

We will now prove the following Lemma:

Lemma 3. : $\Delta$ is the same for $\Phi[s]$ and for $B_{1}(s) \Phi \Pi^{-1}$. Proof 4. Let $B_{1}(s) \Phi \Pi^{-1}$ and $n_{i e}-\partial \hat{\varphi}_{i, j}=\hat{\gamma}_{i}$, for $i=$ $1, \ldots, p-k$

1- In (A.3), each polynomial $\hat{\varphi}_{i, 1}$ of $\hat{\Phi}_{1}[s]$ is given by:

$$
\hat{\varphi}_{i, 1}=-\varphi_{p, p} \frac{\varphi_{i, 1}}{\varphi_{p, 1}},
$$

with $\partial \varphi_{p, p}=n_{p e}$ and $\partial \varphi_{p, 1}=n_{1 e}$. Then $n_{p e}-\partial \hat{\varphi}_{i, j}=$ $n_{1 e}-\partial \varphi_{i, j}$. So $\hat{\gamma}_{1}=\gamma_{1}$. Especially, if we had $\gamma_{1}=\Delta$,

\footnotetext{
1 We could always suppose that $\varphi_{p, 1}$ is normalized as $s^{n_{1 e}}$
} 
this index is transmitted in the first column of $B_{1}(s) \Phi \Pi^{-1}$. But still nothing proves here that $\Delta=\min \left\{\hat{\gamma}_{i}\right\}_{p-k}$.

2 - For the other columns of $\hat{\Phi}_{1}[s], \hat{\varphi}_{i, j}=\varphi_{i, j}-\varphi_{p, j} \frac{\varphi_{i, 1}}{\varphi_{p, 1}}$ and then: $\partial \hat{\varphi}_{i, j} \leq \max \left(\partial \varphi_{i, j}, \partial \varphi_{p, j} \frac{\varphi_{i, 1}}{\varphi_{p, 1}}\right)$.

Then or $\hat{\gamma}_{j}=\min _{i}\left\{n_{j e}-\partial \varphi_{i, j}\right\}_{p-k}=\gamma_{j}$, or $\hat{\gamma}_{j}=$ $\min _{i}\left\{n_{j e}-\partial \varphi_{p, j}-\partial \varphi_{i, 1}+\partial \varphi_{p, 1}\right\}_{p-k}$ : in this last case, as $\partial \varphi_{p, 1}-\partial \varphi_{i, 1} \geq \gamma_{1}$, we have:

$n_{j e}-\partial \hat{\varphi}_{i, j} \geq n_{j e}-\partial \varphi_{p, j}+\gamma_{1}=\gamma_{1}+c$, where $c \geq 0$.

So, $\hat{\gamma}_{j} \geq \min \left\{\gamma_{j}, \gamma_{1}+c\right\}$. If $\gamma_{j}=\Delta=\gamma_{1}$, there may be cancellation of the terms of highest degree of the polynomial and then $\hat{\gamma}_{j}>\Delta$. But, in this case, $\Delta$ is still in the first column, as we have seen in item 1 . The consequence is that, for each column $j$,

$$
\hat{\gamma}_{j} \geq \min \left(\gamma_{j}, \gamma_{1}\right)
$$

and $\Delta=\min \left\{\hat{\gamma}_{i}\right\}_{p-k}$, which ends the proof of Lemma 3 .

\section{A.2 $\Delta$ is the smallest decoupling index of $\Sigma$}

Suppose that, for the $r$ th column of $B_{1}(s) \Phi \Pi^{-1}, n_{r e}-$ $p_{r e}=\Delta$ and $\partial \hat{\varphi}_{s, r}=p_{r e}$. Permute $\left(\right.$ by $\left.\Pi_{r}^{-1}\right)$ the $r$ th and the $(p-k)$ th columns of $B_{1}(s) \Phi[s] \Pi^{-1}$. We obtain

$$
B_{1}(s) \Phi \Pi^{-1} \Pi_{r}^{-1}=\left[\begin{array}{cc}
\hat{\Phi}_{1, r}(s) & (0) \\
\hat{\Phi}_{2, r}(s) & \hat{\Phi}_{3}[s]
\end{array}\right]=\left[\hat{\varphi}_{r_{i, j}}\right]
$$

There exists a biproper transformation $B_{2}(s)$ such that the interactor of the new permuted system is given by $\Phi_{r}[s]=B_{2}(s) B_{1}(s) \Phi[s] \Pi^{-1} \Pi_{r}^{-1}=\left[\hat{\varphi}_{r i, j}\right]$. From (A.6), $B_{2}(s)$ has the following structure:

$$
B_{2}(s)=\left[\begin{array}{cc}
B_{2,1}(s) & (0) \\
B_{2,2}(s) & \mathbb{I}_{k}
\end{array}\right],
$$

where $B_{2,1}(s)$ is a biproper $p-k \times p-k$ matrix such that $B_{2,1}(s) \hat{\Phi}_{1, r}(s) \Pi_{r}^{-1}$ is structured as an interactor. As $B_{2}(s)$ and $B_{2,1}(s)$ are biproper, the maximum degrees of each column $j$ of $B_{2}(s) B_{1}(s) \Phi \Pi^{-1} \Pi_{r}{ }^{-1}$ and of $B_{2,1}(s) \hat{\Phi}_{1, r}[s]$ are respectively equal to $n_{j e}$ and $p_{i e}$. Then $\hat{\varphi}_{r_{p-k, p-k}}=s^{p e}, \hat{\varphi}_{r_{i, p-k}}=0$ for $i=1, \ldots, p-k-1$, and the monomials of degree less than or equal to pe have been canceled by $B_{2,2}(s)$ in the polynomials $\hat{\varphi}_{r, p-k}=0$ for $i=p-k+1, \ldots, p$. So, the decoupling index of the new $(p-k)$ th output, noted $\Delta_{p-k}$ is $\Delta_{p-k}=\Delta$. It is the smallest possible for SRMP from Lemmas 1 and 3 .

Remark 6. From (A.5) and as $B_{2,1}(s)$ is biproper, the new quantities $\hat{\gamma}_{r_{i}}$ computed for the interactor $\Phi_{r}[s]$ are greater than or equal to $\Delta_{p-k}=\Delta$.

\section{A.3 the minimal list of decoupling indices}

Let $\Phi_{r}[s]$ defined in subsection A.2. Note

$$
\tilde{\Phi}[s)=\left[\begin{array}{cc}
\tilde{\Phi}_{1}[s] & (0) \\
\tilde{\Phi}_{2}[s] & \tilde{\Phi}_{3}[s]
\end{array}\right]=\left[\tilde{\varphi}_{i, j}[s]\right] .
$$

where $\tilde{\Phi}_{1}[s]$ has the structure of a $p-k-1 \times p-k-1$ interactor. We apply the same procedure as before with regard to the columns $1,2, \ldots, p-k+1$ of $\Phi_{r}$ : we obtain a second decoupling index $\Delta_{p-k-1} \geq \Delta_{p-k}$ which is as small as possible at this step. This procedure is iterated until obtaining the unique list $\left\{\Delta_{i}\right\}_{(p-k)}=\Delta_{1} \geq \Delta_{2} \geq$ $\ldots \geq \Delta_{p-k}=\Delta$ of the $p-k$ integers. The minimality comes from the fact that at each iteration $i$, the increase of infinite structure $\Delta-i$ is the smallest that we can obtain by permutation of the outputs of $\Sigma$. Then this list "minore" all the other lists at the sense of Definition 2 .

\section{REFERENCES}

Commault, C., Dion, J., Descusse, J., Lafay, J.F., and Malabre, M. (1986). New decoupling invariants: the essential orders. Int. J. of Control, vol. 44(no.3), 689700.

Brunovski, P. (1970). A classification of linear multivariable system. Kybernetika, vol. 6(no.3), 173-188.

Dion, M. and Commault, C. (1988). The minimal delay decoupling problem: feedback implenentation with stability. SIAM J. Contr Optimiz., vol. 26, 66-82.

Falb, P.L. and Wolovich, W.A. (1967). Decoupling in the design and synthesis of multivariable control systems. IEEE Trans. on Automat. Contr., vol. 12(no.6), 651669.

Hautus,M.L.J. and Heymann,M. (1978). Linear feedback, an algebraic approach. SIAM J. Contr. Optimiz., vol. 7, 50-63.

Herrera H, A.N. (1991). Sur le découplage des systèmes linéaires par des lois statiques non régulières. Thèse de doctorat, Université de Nantes, Ecole Centrale de Nantes.

Herrera H, A.N. (1992). Static realization of dynamic precompensators. IEEE Trans. on Automat. Contr., vol. 37(no.2), 1391-1394.

Herrera H, A.N. and Lafay, J.F. (1993). New results about the Morgan's problem. IEEE Trans. on Automat. Contr., vol. 38(no.12), 1834-1838.

Herrera H, A.N., Lafay, J.F., and Zagalak, P. (1997). A semi-canonical form for a class of right invertible linear systems. Automatica, vol. 33(no.2), 269-271.

Lafay, J.F., Zagalak, P., and Herrera H, A.N. (1992). Reduced form of the interactor matrix. IEEE Trans. on Automat. Contr., 37(11), 1778-1782.

Lafay, J-F.(2013). .Minimal decoupling indices for linear systems In IFAC joint TDS-SSSC-FDA 2013 Conference. february 4-6, Grenoble France.

Loiseau, J.J. (1988). Sur la modification de la structure à l'infini par retour d'état statique. SIAM J. Contr. and Optimiz., vol. 26, 251-273.

Morgan Jr, B. (1964). The synthesis of linear multivariable systems by state feedback. J.A.C.C. 64, 468-472.

Morse, A.S. (1973). Structural invariants of linear multivariable systems. SIAM J. Contr. Optimiz., vol. 11(no.3), 446-465.

Wolovich, W.A. and Falb, P.L. (1976). Invariants and canonical forms under dynamic compensation. SIAM J. Contr. Optimiz., vol. 14, 996-1008.

Zagalak, P., Eldem, V., and Ozcaldiran, K. (1998). On a special case of the Morgan problem. In 5th Conference IFAC System Structure and Control, volume 1, 169-174. Nantes France. 